\title{
Tecnologías para el desarrollo de nuevos fármacos
}

La tarea científica del Químico Farmacéutico en el desarrollo de nuevos productos para prevenir y combatir enfermedades ha sido y es muy importante, así como también lo es la publicación de estos esfuerzos en favor del nuevo conocimiento. Por ello, se hace imprescindible la continuidad de nuestra revista Ciencia e Investigación, que es verdadera expresión del avance científico y la permanente actualización de conocimientos en nuestra Facultad. Bien se ha dicho "trabajo de investigación que no se publica NO EXISTE".

Como escribía el Dr. Fortunato Carranza, Decano de la Facultad de Farmacia y Bioquímica y primer Rector farmacéutico de la Universidad Nacional Mayor de San Marcos: "Con un interés en la mente y una lupa en la mano el investigador científico interroga a la naturaleza para extraerle sus secretos y convertirlos en medicamentos" para describir las responsabilidades del Químico Farmacéutico en sus esfuerzos por mejorar día a día la búsqueda de nuevos medicamentos y elevar su calidad y eficacia.

Desde hace muchos años, el modelo para comprobar la bondad y eficacia del medicamento ha sido en su primera fase el trabajo in vitro, para luego pasar a la etapa experimental induciendo la enfermedad que se estudia en animales de experimentación, ratones, ratas, perros, cuyes, conejos, etc. En ellos se determina el efecto terapéutico del nuevo medicamento en estudio. Nuestra hemeroteca conserva numerosas tesis realizadas con este fin y utilizando este procedimiento.

Tres investigadores obtuvieron el Premio Nobel de Medicina en el 2007 por haber implementado exitosamente la tecnología del ratón Knockout o ratón $\mathrm{KO}$, en el que se logró anular la actividad y funciones de un gen determinado, permitiendo así su estudio y descripción de cómo es que aparecen las enfermedades. De allí que estos ratones KO pueden considerarse como organismos modelo para desarrollar y comprobar fármacos.

Uno de los tres galardonados con el Premio Nobel, Mario Capecchi, estuvo en Lima invitado a participar en un evento científico organizado por el Colegio Médico del Perú. El doctor Capecchi ofreció 4 interesantes conferencias con temas de su especialidad y una charla final referida a acontecimientos importantes de su vida. Hoy día este procedimiento genético se ha facilitado y ampliado enormemente al aplicarse la novedosa y sencilla prueba de edición genética CRISPR/Cas9.

En este decenio, y continuando el imparable progreso en este tema de modelamiento para desarrollar medicamentos, se ha conseguido obtener organoides humanos a partir de células madre pluripotentes inducidas, que Shinya Yamanaka logró mediante un diseño elegante, obtener células madre a partir de células adultas de piel (no embrionarias) y que le valió el Premio Nobel de Medicina 2012.

Según el Dr. Alberto Muñoz (BJS): "Se definen los organoides como cultivos tridimensionales derivados de células madre que reproducen parcialmente las características de sus tejidos de origen".

El proceso para obtener un organoide humano requiere de su cultivo tridimensional y un minireactor con agitador. A la fecha se han obtenido diversos organoides derivados de tejidos de órganos huma-

Citar como:

Amiel J. Tecnologías para el desarrollo de nuevos fármacos [Editorial]. Ciencia e Investigación 2019 22(1):3-4.

( ) Los autores. Este artículo es publicado por la Revista Odontología Sanmarquina de la Facultad de Odontología, Universidad Nacional Mayor de San Marcos. Este es un artículo de acceso abierto, distribuido bajo los términos de la licencia Creative Commons Atribucion - No Comercia_Compartir Igual 4.0 Internacional. (http://creativecommons.org/licenses/by-nc-sa/4.0/) que permite el uso no comercial, distribución y reproducción en cualquier medio, siempre que la obra original sea debidamente citada. 
nos que por su tamaño muy pequeño se denominan organoides, entre ellos se ha creado, según la literatura científica, organoides de cerebro, intestinos, riñones, colon, hígado, esófago y estómago. Se ha publicado que organoides derivados de pacientes podrían ayudar a personalizar el tratamiento del cáncer gastrointestinal. Con ellos se pueden realizar diversas pruebas que son más simples que los estudios clínicos que requieren 4 fases en el hombre, además no conllevan los riesgos que esta labor final requiere y también se podrá obtener mucha información a bajo costo.

Para todas estas informaciones científicas se requiere de CIENCIA E INVESTIGACIÓN, la cual, debe esperarse, habrá de recibir la colaboración de los investigadores y estudiosos profesores de nuestra Facultad.

Dr. José Amiel Pérez

Profesor Emérito y Pas Rector

Universidad Nacional Mayor de San Marcos 